\title{
Simultaneous Comparison of Electrocardiographic Imaging and Epicardial Contact Mapping in Structural Heart Disease
}

Adam J. Graham MRCP $*$, Michele Orini PhD ${ }^{a, b} *$, Ernesto Zacur PhD ${ }^{c}$, Gurpreet Dhillon MRCPa Holly Daw MSca, Niel T. Srinivasan MRCPa, Jem D. Lane PhD MRCPa, Alex Cambridge BSc ${ }^{a}$, Jason Garcia BSc a, Nanci J O'Reilly MBBSa , Sarah Whittaker-Axon BSc ${ }^{a}$, Peter Taggart MD DSc ${ }^{d}$, Martin Lowe PhD FRCPa, Malcolm Finlay PhD MRCPa, Mark J Earley MD FRCPa, Antony Chow MD MRCP FHRSa, Simon Sporton MD FRCPa, Mehul Dhinoja MD MRCP FHRSa, Richard J Schilling MD MRCP FHRSa, Ross J Hunter MRCP FHRS PhDa, Pier D. Lambiase PhD FRCP FHRS ad

a. Barts Heart Centre, Barts Health NHS Trust, West Smithfield, London EC1A 7BE, UK

b. Department of Mechanical Engineer, University College London, London, UK

c. Institute of Biomedical Engineering, University of Oxford, Oxford, UK.

d. Institute of Cardiovascular Science, University College London, Gower Street, London WC1E 6BT, UK

* Equal contributors

Address for correspondence:

Professor Pier D. Lambiase

UCL Institute of Cardiovascular Science \& Cardiology Dept, Barts Heart Centre, Barts Health NHS Trust, West Smithfield, London, EC1A 7BE, UK

Email: p.lambiase@ucl.ac.uk

Tel: 02037658647

RUNNING TITLE: Simultaneous ECGI vs Contact mapping comparison 


\section{Abstract}

Background: The accuracy of ECG Imaging (ECGI) in structural heart disease remains uncertain. This study aimed to provide a detailed comparison of ECGI and contact-mapping system (CARTO) electrograms.

Methods: Simultaneous epicardial mapping using CARTO (Biosense-Webster, CA, USA) and ECGI (Cardiolnsight ${ }^{\mathrm{TM}}$ ) in 8 patients was performed to compare electrogram morphology, activation (AT) and repolarization (RT) times. Agreement between AT and RT from CARTO and ECGI was assessed using Pearson's correlation coefficient, $\rho_{A T}$ and $\rho_{R T}$, root mean square error, $E_{A T}$ and $E_{R T}$, and Bland-Altman plots.

Results: After geometrical co-registration, 711 (439-905) (median, first-third quartiles) ECGI and CARTO points were paired per patient. Activation time maps showed $\rho_{A T}=0.66(0.53-0.73)$ and $E_{A T}=24(21-32) \mathrm{ms}, \mathrm{RT}$ maps showed $\rho_{R T}=0.55(0.41-0.71)$ and $E_{R T}=51$ (38-70) $\mathrm{ms}$. The median correlation coefficient measuring the morphological similarity between the unipolar electrograms was equal to $0.71(0.65-$ $0.74)$ for the entire signal, $0.67(0.59-0.76)$ for QRS complexes and $0.57(0.35-0.76)$ for T-waves. Local activation map correlation, $\rho_{A T}$, was lower when default filters were used (0.60 (0.30-0.71), P=0.053). Small misalignment of the ECGI and CARTO geometries (below $\pm 4 \mathrm{~mm}$ and $\pm 4 \mathrm{deg}$ ) could introduce variations in the median $\rho_{A T}$ up to $\pm 25 \%$. Minimum distance between epicardial pacing sites and the region of earliest activation in ECGI was $13.2(0.0-28.3) \mathrm{mm}$ from 25 pacing sites with stimulation to QRS interval $<40 \mathrm{~ms}$.

Conclusions: This simultaneous assessment demonstrates that ECGI maps activation and repolarization parameters with moderate accuracy. ECGI and contact electrogram correlation is sensitive to electrode apposition and geometric alignment. Further technological developments may improve spatial resolution. 
Keywords: Contact mapping, ECG-Imaging, Cardiolnsight, Activation and Repolarization Maps, Unipolar Electrograms, Ventricular Tachycardia 


\section{Introduction}

Electrocardiographic imaging (ECGl) utilizes body surface potentials and heart-torso geometries to reconstruct epicardial unipolar electrograms. This is achieved applying an "inverse solution" and provides insight into the electrophysiological substrate customarily only delineated with invasive contact electro-anatomical mapping (EAM) 1,2 .

The ability to delineate whole tachycardia circuits using a single beat has important implications for haemodynamically-unstable ventricular tachycardias (VTs), which cannot be easily mapped using EAM ${ }^{3}$. Furthermore, this technology could facilitate risk stratification in primary prevention ICD candidates ${ }^{4,5}$. Initial experimental validation of the methodology utilizing a tank-torso model showed its ability to image cardiac activation and repolarization ${ }^{2,6,7}$, which was confirmed in open chest canines versus contact electrogram data ${ }^{8}$. Human work during cardiac surgery showed promising results although the contact and ECGI maps were not acquired simultaneously ${ }^{9}$. This has culminated in the use of the system for ablation of focal ectopy, VT, atrial fibrillation ${ }^{10,11}$ and non-invasive ablation of ventricular arrhythmias with radiotherapy ${ }^{12,13}$.

However, despite the range of clinical applications there has been no direct quantitative simultaneous comparison to contact electrogram data in the intact human heart. Simultaneous collection of epicardial contact and body surface ECGI data in canine and porcine models showed moderate correlation for activation time (AT) and repolarization time $(\mathrm{RT}){ }^{14,15}$. In humans, a using a 120 lead system, simultaneous contact electrical data demonstrated variable accuracy for locating epicardial pacing sites and qualitatively assessed ventricular activation sequences ${ }^{16}$. Non- 
simultaneous mapping recently showed ECGI reconstruction of activation sequences was poor during sinus rhythm with a narrow QRS complex ${ }^{17}$.

This study set out to prospectively compare simultaneously-recorded epicardial contact electrograms with reconstructed epicardial electrograms from a 252 electrode ECGI system (Cardiolnsight ${ }^{\mathrm{TM}}$, Medtronic, MN, USA) in the intact human heart.

\section{Methods}

The authors declare that all supporting data are available within the article and its online supplementary files. The row data that support the findings of this study are available from the corresponding author upon reasonable request. Eight patients (5 male, 3 female), aged $45.9 \pm 15.5$ years undergoing epicardial catheter ablation of structurally abnormal heart VT were studied with ECGI mapping during ablation. All patients were scheduled for a catheter ablation procedure on clinical grounds and gave their informed consent to participate in the research study. The study was approved by the National Research Service Committee, London (14/LO/0360).

\section{Clinical Procedure}

Procedures were performed with the patient under general anaesthetic (GA). Endocardial access was obtained under ultrasound guidance using Seldinger technique via the right femoral vein $+/$ - right femoral artery. A sub-xiphisternal puncture using a Tuohy needle, with fluoroscopic guidance, was used to access the epicardial space ${ }^{18}$. An Electro-anatomical map (EAM) (CARTO, Biosense-Webster, CA, USA) of the epicardial surface was created during right ventricular pacing in six patients, biventricular pacing in one patient and atrial pacing in another. This was to ensure stable rhythms for electrogram data collection. For the latter two patients pacing 
modalities were chosen to improve haemodynamic status during mapping. A multipolar catheter (Pentarray or Decapolar, Biosense-Webster, CA, USA) was used for all cases. Unipolar electrograms were collected from all points during EAM creation with bandpass filters set at $0.5 \mathrm{~Hz}$ to $500 \mathrm{~Hz}$ and a sampling frequency of $1000 \mathrm{~Hz}$. Pacing was performed from multiple locations on the epicardium at $<10 \mathrm{~mA}$.

\section{ECGI recordings}

Prior to catheter ablation, a 252 electrode vest (Cardiolnsight ${ }^{\mathrm{TM}}$, Medtronic, MN, USA) was fitted for recording of body surface potentials (sampling rate $1000 \mathrm{~Hz}$ ) and remained in situ until conclusion of the procedure. A non-contrast axial CT scan with $3 \mathrm{~mm}$ slice thickness was performed up to four hours before the procedure. Patientspecific epicardial geometry was created using the EcVue system (Medtronic, MN, USA) with data from the CT and body surface potentials. Epicardial unipolar electrograms were computed over approximately 1400 epicardial points covering both ventricles using both unfiltered and filtered (low pass $50 \mathrm{~Hz}$ ) data, with those over the atrioventricular valves manually excluded. A full aortic mesh, comprising the ascending, arch and descending portions, was created in patients with arterial access. If arterial access was not obtained a detailed geometry of the right ventricular outflow tract (RVOT), inferior vena cava (IVC) and superior vena cava (SVC) was generated.

\section{Data Analysis}

Unipolar electrograms from CARTO and ECGI were independently analysed with bespoke software (Matlab, The Mathworks Inc., MA, USA). After pacing artefact removal, signals were band-pass filtered between 0.5 and $80 \mathrm{~Hz}$ for AT measurement and between 0.5 and $20 \mathrm{~Hz}$ for RT measurement. Activation time was measured as 
the time of the steepest signal downslope ( $\mathrm{dV} / \mathrm{dt}$ min $)$ during the QRS complex and RT the time of steepest upslope ( $\left.d \mathrm{~V} / \mathrm{dt}_{\max }\right)$ during the $\mathrm{T}$-wave ${ }^{19,20}$. All signals were carefully reviewed and semi-automatically corrected if needed as in previous studies 21,22 .

Co-registration of EAM and ECGI geometries was performed semi-automatically with bespoke software (Matlab, The Mathworks Inc., MA, USA). As Figure 1 shows, accuracy was achieved by simultaneous alignment of all prominent anatomical geometries including the Aorta (4 patients) and IVC and RVOT (in 4 patients). The optimal co-registration was visually determined by two experts independent of subsequent analysis. After co-registration, for each point belonging to the ECGI geometry, the closest point belonging to the CARTO geometry was found and the two points were paired for comparison. ECGI and CARTO points were paired only if their Euclidean distance was lower than $\mathrm{D}=8 \mathrm{~mm}$. To reduce the effect of outliers, spatial smoothing was performed by averaging AT and RT of points contained within a $D=8$ $\mathrm{mm}$ radius. The analysis was repeated for $D$ ranging from 5 to $14 \mathrm{~mm}$ to assess the impact of spatial smoothing on the results. To assess the sensitivity of our results to co-registration between ECGI and CARTO geometries, the analysis was repeated after applying small changes to the optimal co-registration. In total, the analysis was repeated 4096 times per patient consistent with all possible configurations obtained by applying a shift of $-4,-2,+2$ and $+4 \mathrm{~mm}$ and a rotation of $-4,-2,+2$ and +4 degrees along and around the three major axes.

To assess spatial resolution of the localization of earliest sites of epicardial activation, the minimum Euclidean distance between the pacing pole (projected onto the ECGI ventricular geometry) and the region of earliest activation (within the first $5^{\text {th }}$ percentile) 
in the ECGI map was measured. The time from stimulus to QRS (S-QRS) was calculated for each pacing beat. Beats were then separated into those with a S-QRS of $<40 \mathrm{msec}$ and $>40 \mathrm{msec}$.

\section{Statistical analysis}

Data distribution is described by median (first-third quartiles). Statistical differences were assessed using the Wilcoxon rank sum test for unpaired comparisons and the Wilcoxon signed rank test for paired comparisons. Threshold for statistical significance was 0.05 . The morphological similarity between each pair of signals was assessed using Pearson's correlation coefficient. The agreement between activation and repolarization sequences between the two modalities was quantified with the root mean square error, Pearson's correlation coefficient and Bland-Altman plots. Interpatient correlations between e.g. the correlation coefficient for AT or RT maps and QRS duration, QRS amplitude or number of electrodes in contact with the body surface were assessed using Spearman's correlation coefficient. Data and statistical analyses were conducted in MATLAB, MathWorks.

\section{Results}

Eight patients were studied. Five were elective procedures with the remaining three in-patient emergency procedures. Baseline characteristics can be seen in Table 1. The prevalence of ARVC cases accounted for by the epicardial nature of this condition.

\section{Activation and repolarization maps}

3790 (1845-6022) (median, Q1-Q3) and 1385 (1362-1395) unipolar signals per patient from CARTO and ECGI, respectively, were used for the analysis. Of these, 711 (439- 
905) per patient were paired and used for comparison. Figures 2 and 3 show examples of AT and RT maps produced by the two systems for comparison, while numerical results are shown in Table 2. Visually, there was consistency between ECGI and EAM maps. Correlation coefficient measuring the similarity of AT and RT sequences was equal to $0.66(0.53-0.73)$ for $\mathrm{AT}$ and $0.55(0.41-0.72)$ for $\mathrm{RT}$, while root mean square error was equal to 24 (21-35) ms for AT and 51 (38-70) ms for RT. Seven of eight patients had a correlation coefficient for AT sequence higher than 0.5. Scatter-plots and Bland-Altman plots showing inter-modality agreement for AT and RT per each patient are shown in Supplementary Figures 1-8. The correlation coefficient for AT maps showed moderate correlation with QRS duration $(\rho=0.52)$, suggesting that slower AT sequences can be non-invasively mapped more reliably, and with mean QRS amplitude of the 12 leads ECG ( $\rho=0.65$, excluding the one patient with atrial pacing) suggesting that structural heart disease and low signal amplitude may reduce mapping accuracy. The number of body surface potentials included in the computation of the inverse problem also showed moderate correlation with correlation coefficient for AT maps $(\rho=0.40)$, indicating that care should be taken in maintaining good contact between the ECGI vest and patient's torso. The correlation coefficient for AT maps decreased from $0.66(0.53-0.73)$ to $0.60(0.30-0.7), P=0.053$, if the body surface potentials were low-pass filtered using the by default filter setting of the Cardiolnsight ${ }^{\mathrm{TM}}$ system prior to reconstructing the epicardial potentials.

\section{Signal morphology}

The correlation coefficient measuring the morphological similarity between unipolar electrograms recorded with contact mapping and computed with ECGI was equal to $0.71(0.65-0.74)$ when considering the entire duration of the signal, and equal to 0.67 
(0.59-0.76) within the QRS complex and $0.57(0.35-0.76)$ for the T-wave (see Table 2). Seven of eight patients had a morphological correlation coefficient for the QRS complex higher than 0.5 . These results were not significantly different when computed using pre-filtered data. Supplementary Figures 9-16 show the distribution of the correlation coefficient and representative electrogram examples going from best to worst correlation. There was a marked intra-patient variability in the correlation coefficients measuring morphological similarity between recorded and computed electrograms, with median interquartile range equal to 0.67 .

\section{Localization of earliest sites of activation}

In figure 4 examples of localisation of pacing sites on the EAM to the area of earliest activation on ECGI map are shown for three representative patients. The white circle represents the pacing site projected onto the ECGI geometry. Table 3 shows distance for pacing site accuracy in all patients.

Overall, the distance from $n=46$ epicardial pacing sites to the corresponding areas of earliest activation was 20.7 (9.6-33.2) $\mathrm{mm}$. This was significantly lower for the $\mathrm{n}=25$ pacing sites for which local capture was confirmed by a short stim-to-QRS interval, with distance equal to $13.2(0.0-28.3) \mathrm{mm}$ for stim-to-QRS interval $\leq 40 \mathrm{~ms}$ versus 32.6 $(21.5-45.8) \mathrm{mm}(\mathrm{P}<0.001)$ for stim-to-QRS interval $>40 \mathrm{~ms}$.

\section{Agreement between EAM and ECGI in low-voltage regions}

Low-voltage regions were defined as cardiac sites for which EAM registered a bipolar signal amplitude $<0.5 \mathrm{mV}$. Indices of agreement between EAM and ECGI calculated in low voltage regions were not different to those calculated in normal voltage regions (Supplementary Table 1). Pacing sites were considered as belonging to a low-voltage 
region if the median bipolar amplitude recorded with EAM within a search radius equal to $4 \mathrm{~mm}$ was $<0.5 \mathrm{mV}$. The distance between pacing sites and the regions of earliest activation in ECGI was not different when comparing pacing sites in low versus normal voltage regions (Supplementary Table 1).

\section{Effects of anatomical co-registration}

Parameter $D$, which corresponds to the minimum distance used for pairing cardiac sites from EAM and ECGI maps and determines the amount of spatial smoothing did not have a significant impact on the results (Supplementary Fig. 17). On the other hand, small variations in the anatomical co-registration may have an impact on the results. Although on average the agreement between ECGI and CARTO AT maps $\left(\rho_{A T}\right)$ did not change after applying small changes to the optimal anatomical coregistration (Supplementary Table 2), the selection of the configuration that for each patient maximises or minimises $\rho_{A T}$ (see Supplementary Figure 18-25 for comparison) would have resulted in a $\pm 25 \%$ variation of the median $\rho_{A T}$ (Supplementary Table 1 ).

\section{Discussion}

This is the first quantitative comparison of reconstructed electrograms using ECGI versus simultaneously recorded contact epicardial data, in the intact human heart. The main findings are: Morphological correlation between recorded and computed unipolar electrograms show median correlation coefficient per patient was equal to $0.71(0.65-0.74)$; AT maps showed a correlation of $\rho_{A T}=0.66(0.53-0.73)$ and error of $E_{A T=24}(21-32) \mathrm{ms} ; \mathrm{RT}$ maps showed $\rho_{R T}=0.55(0.41-0.71)$ and $E_{R T}=51$ (38-70) ms. Minimum distance between epicardial pacing sites and the region of earliest activation 
in ECGI was $13.2(0.0-28.3) \mathrm{mm}$ for pacing sites where a short stim-to-QRS interval confirmed local capture. This quantitative assessment is sensitive to anatomical coregistration.

Overall these data suggest that the accuracy of the non-invasive mapping system may not provide sufficient resolution to guide radio frequency ablation of ventricular arrhythmias but could shorten, and potentially improve the efficiency of ablation procedures by allowing rapid targeting for contact mapping focal ectopics or in situations of haemodynamic instability.

The correlations reported in this study are similar to those demonstrated in proof-ofprinciple studies. Initial experiments using the tank-torso model found a correlation coefficient (CC) of 0.81 for $\mathrm{AT}^{6}$ but the tank-torso model does not take into account motion artefact during respiration or the effect of lung tissue between the epicardium and body surface electrodes ${ }^{23}$.

A CC of 0.72 for AT was found in cardiac surgery patients using consecutive and not simultaneously recorded data ${ }^{9}$. More recently, a canine model, under closed-chest conditions, found a median CC of 0.73 for AT ${ }^{14}$. While these data show higher correlation than 0.66 we observed, our data are the first simultaneously-recorded quantitative clinical data taken during an ablation procedure. Our results are comparable to a simultaneous ECGI epicardial sock closed chest porcine study showing mean CCs for reconstructed epicardial potential distributions ranged from $0.60 \pm 0.08$ to $0.64 \pm 0.07$ and general activation spread median CC $0.72-0.78$ for activation time maps after spatio-temporal smoothing ${ }^{15}$.

Our results contrast with recently published data comparing ECGI and EAM in 55 patients, where AT was found to be largely inaccurate (CC $0.03 \pm 0.43$ ) ${ }^{17}$. Mapping 
was performed mainly during sinus rhythm, with better correlation seen during paced rhythms. The study used the commercially available system for production of AT maps without curation of individual electrogram timing measurements. The lower values could reflect the methods used to create ECGI maps and the fact that bipolar electrograms from EAM were utilised without direct cross-correlation with the corresponding unipolar contact signals which could be a further source of erroneous measurement. We used bespoke software to analyse the electrograms along with manual editing. In our experience significant editing of the electrograms is required to ensure accurate annotation of AT in both contact and non-contact mapping data. We also found moderate association between AT maps correlation and body surface QRS duration $(\rho=0.52)$. This suggests that ECGI maps slower myocardial activation wavefront progression more accurately and has important implications for mapping during narrow QRS rhythms where recent data indicated reduced accuracy of identifying epicardial breakthroughs and location of lines of block by comparison with high density epicardial mapping ${ }^{17}$. Narrower QRS complexes reflect more rapid myocardial activation utilizing the Purkinje network and are not representative of the slower activation of ventricular arrhythmias and epicardial pacing. Epicardial recruitment would be expected to be slower to enable more accurate mapping by ECGI since larger myocardial segments will be activated simultaneously within the temporal-spatial resolution of the system. Indeed, recent success in targeting of ablation resistant VT circuits using ECGI mapping indicates that resolution may be sufficient for delivering stereotactic radiation for non-invasive VT ablation. VT episodes decreased from $119(4-292)$ to $3(0-31)$ in 19 patients at 6 months post treatment ${ }^{12,13}$. This novel methodology has thus far been performed in a single centre and further 
research will be needed into both its long-term efficacy and the role played by ECGI to guide this ablation modality.

Filter settings on the ECGI system exert significant effects, as local activation map correlation, $\rho_{A T}$, was significantly lower when default filters were used. This is important as commercially-available systems currently automatically apply a low pass filter of $50 \mathrm{~Hz}$. Optimization of the number of electrodes in good contact with the body surface is also needed as utilizing fewer electrodes may confound results.

To the best of our knowledge, this is the first attempt in humans to quantify the relationship of RT between recorded and reconstructed electrograms. Reconstruction of spatial variation of $R T$ is an important feature of ECGI in the potential risk stratification of arrhythmic risk ${ }^{24}$. Human torso models have shown that myocardial repolarization was accurately reflected by ECGI ${ }^{8,25}$. Our results can be compared to the afore-mentioned canine model which showed CC of $0.6^{14}$. Correlations are lower than those for AT. This is expected given the smaller amplitude of the $T$ wave, increasing susceptibility to noise and electrogram smoothing introduced by the inverse solution algorithm. Furthermore, as reported in direct porcine study, although the overall electrogram CC between measured and reconstructed epicardial electrograms was $\approx 0.7$, the interquartile ranges were wide. That is, while agreement was reasonable in $\approx 50 \%$ of cases, it was much less in the remainder ${ }^{15}$.

Indices of agreement between ECGI and EAM were not different in low voltage as compared to normal voltage regions. This may be explained by the fact that in fibrotic tissue the local component of the unipolar electrogram is small or non-existent and the signal is essentially composed of far-field potential ${ }^{20}$, which may be measured by both contact and non-contact systems with moderate correlation. 


\section{Localisation of pacing sites}

The minimum distance between epicardial pacing sites and the region of earliest AT in ECGI was $13.2 \mathrm{~mm}(0.0-28.3)$ from 25 pacing sites with stim-to-QRS $<40 \mathrm{~ms}$. The distances were significantly greater with stim-to-QRS $>40 \mathrm{msec}$. Other research has shown resolution for locating pacing of around $10 \mathrm{~mm}{ }^{9,26} 27$ with a more contemporary study in humans of $<10 \mathrm{~mm}$ for both RV and LV endocardial pacing ${ }^{28}$. The only other study to perform a simultaneous epicardial pacing in humans found results in keeping with ours ${ }^{16}$ with a decreased distance from pacing site to area of earliest activation when the stim-to-QRS was $<40 \mathrm{msec}$. The distance between pacing sites and the regions of earliest activation in ECGI was not greater in regions were EAM registered low bipolar voltage. As pacing from a low voltage region was not clearly associated with a long stimulus-to-QRS interval, this may be due to inaccuracies of bipolar amplitude to delineate scar ${ }^{29,30}$.

\section{Geometric Alignment}

Small movements in the co-registered geometries can have large effects on correlation. Considering the most extreme cases, shifts of only $\pm 4 \mathrm{~mm}$ and rotations within \pm 4 degrees can results in a variation of the ECGI-EAM AT correlation up to $\pm 25 \%$. This may confound studies examining the accuracy of ECGI and should be considered in future research protocols to ensure optimal co-registration of geometries.

\section{Limitations}

This study compared contact EAM to ECGI provided by the Cardiolnsight ${ }^{\mathrm{TM}}$ system as used in the cathlab during VT catheter ablation and results may not be easily extended to other ECGI methods ${ }^{31}$. This study focused on epicardial mapping during 
pacing and hence cannot be applied to sinus rhythm where activation sequences and breakthroughs from the endocardium may differ. Pacing was used to ensure stable activation during sequential mapping and every precaution was taken to collect captured beats. However, the EAM consists of many beats collected over a few minutes and ECGI uses a single beat. Although all beats were carefully aligned offline with custom software and manually checked, variation may occur in AT and RT over the course of this period. Recent studies have demonstrated that repeated mapping with different catheters ${ }^{32}$ or even with the same catheter but different activation wavefronts ${ }^{29}$ may introduce significant differences in the delineation of the arrhythmogenic substrate. In this sense, it is not surprising to register differences between EAM and ECGI and our use of mapping catheters with slightly different configuration and intra-electrode distance (Pentarray and Decapolar) may have affected the comparison between EAM and ECGI data.

Only one full EAM was produced during pacing, which was delivered from the RV apex in all except 2 patients. Further studies should assess the effect of different pacing sites and wave-front directions on the agreement between EAM and ECGI.

Geometric alignment of the EAM and CT geometries is challenging but was optimised by using fixed anatomical landmarks as well as ensuring no geometric shifts on CARTO during data collection. However, as demonstrated by our in-depth analysis, EAM-ECGI comparison is intrinsically sensitive to small variations in the anatomical co-registration. This could also have affected the distance measurements garnered from localisation of the pacing points. EAM and ECGI systems estimate the reference potential of unipolar electrogram in a slightly different way, which may have reduced the morphological correlation. This study did not focus on the accuracy of ventricular arrhythmia localisation which is the subject of ongoing work. 


\section{Conclusions}

There is a moderate correlation between reconstructed electrograms recorded using ECGI and contact unipolar electrograms recorded from the epicardium during catheter ablation of ventricular tachycardia in patients with structural heart disease. ECGI and contact electrogram correlations are sensitive to electrode apposition and geometric alignment. Further technological developments may improve spatial resolution and electrogram correlations.

\section{Disclosures}

None

\section{Sources of Funding}

Dr Adam Graham is supported by Barts Charity. Dr Michele Orini is supported by British Heart Foundation (BHF) grant PG/16/81/32441. Prof P. Lambiase is supported by University College London Hospitals Biomedicine National Institute of Health Research (UCLH Biomedicine NIHR) and Barts British Research Centre (BRC). The study was in part supported by a Medtronic External Research Program grant. 


\section{References}

1. Oster HS, Taccardi B, Lux RL, Ershler PR, Rudy Y.

Electrocardiographic Imaging. Circulation [Internet]. 1998 [cited 2018 Dec 4];97:1496-1507. Available from:

https://www.ahajournals.org/doi/10.1161/01.CIR.97.15.1496

2. Burnes JE, Taccardi B, Rudy Y. A noninvasive imaging modality for cardiac arrhythmias. Circulation. 2000;102:2152-2158.

3. Dubois R, Shah AJ, Hocini M, Denis A, Derval N, Cochet H, Sacher F, Bear L, Duchateau J, Jais P, Haissaguerre M. Non-invasive cardiac mapping in clinical practice: Application to the ablation of cardiac arrhythmias. J Electrocardiol [Internet]. 2015;48:966-974. Available from:

https://linkinghub.elsevier.com/retrieve/pii/S0022073615002770

4. Rudy Y. Noninvasive Electrocardiographic Imaging of Arrhythmogenic Substrates in Humans. Circ Res [Internet]. 2013;112:863-874. Available from:

https://www.ahajournals.org/doi/10.1161/CIRCRESAHA.112.27931 5

5. Zhang J, Cooper DH, Desouza KA, Cuculich PS, Woodard PK, Smith TW, Rudy Y. Electrophysiologic Scar Substrate in Relation to VT: Noninvasive High-Resolution Mapping and Risk Assessment with ECGI. PACE - Pacing Clin Electrophysiol [Internet]. 2016 [cited 
2018 Apr 19];39:781-791. Available from:

http://doi.wiley.com/10.1111/pace.12882

6. Ghosh S, Rudy Y. Accuracy of Quadratic Versus Linear Interpolation in Noninvasive Electrocardiographic Imaging (ECGI). Ann Biomed Eng [Internet]. 2005;33:1187-1201. Available from: http://link.springer.com/10.1007/s10439-005-5537-x

7. Ramanathan C, Jia P, Ghanem R, Ryu K, Rudy Y. Activation and repolarization of the normal human heart under complete physiological conditions. Proc Natl Acad Sci [Internet]. 2006;103:6309-6314. Available from:

http://www.pnas.org/cgi/doi/10.1073/pnas.0601533103

8. Burnes JE, Ghanem RN, Waldo AL, Rudy Y. Imaging dispersion of myocardial repolarization, I: Comparison of body-surface and epicardial measures. Circulation [Internet]. 2001;104:1299-1305. Available from:

https://www.ahajournals.org/doi/10.1161/hc3601.094276

9. Ghanem RN, Jia P, Ramanathan C, Ryu K, Markowitz A, Rudy Y. Noninvasive electrocardiographic imaging (ECGI): Comparison to intraoperative mapping in patients. Heart Rhythm [Internet]. 2005;2:339-354. Available from: http://www.scopus.com/inward/record.url?eid=2-s2.015944376227\&partnerlD=40\&md5=9208992b070cf5714680ab8aa 


\section{9ca91}

10. Jamil-Copley S, Bokan R, Kojodjojo P, Qureshi N, Koa-Wing M, Hayat S, Kyriacou A, Sandler B, Sohaib A, Wright I, Davies DW, Whinnett Z, Peters NS, Kanagaratnam P, Lim PB. Noninvasive electrocardiographic mapping to guide ablation of outflow tract ventricular arrhythmias. Heart Rhythm. 2014;11:587-594.

11. Yamashita S, Shah AJ, Mahida S, Sellal J-M, Berte B, Hooks D, Frontera A, Jefairi N Al, Wielandts J-Y, Lim HS, Amraoui S, Denis A, Derval N, Sacher F, Cochet H, Hocini M, Jaïs P, Haïssaguerre M. Body Surface Mapping to Guide Atrial Fibrillation Ablation. Arrhythmia Electrophysiol Rev. 2015;4:172-6.

12. Cuculich PS, Schill MR, Kashani R, Mutic S, Lang A, Cooper D, Faddis M, Gleva M, Noheria A, Smith TW, Hallahan D, Rudy Y, Robinson CG. Noninvasive Cardiac Radiation for Ablation of Ventricular Tachycardia. N Engl J Med [Internet]. 2017;377:23252336. Available from:

http://www.nejm.org/doi/10.1056/NEJMoa1613773

13. Robinson CG, Hugo GD, Lang A. Phase I/II Trial of Electrophysiology-Guided Noninvasive Cardiac Radioablation for Ventricular Tachycardia. Circulation [Internet]. 2018;1-9. Available from: https://www.clinicaltrials.gov/.

14. Cluitmans MJM, Bonizzi P, Karel JMH, Das M, Kietselaer BLJH, de 
Jong MMJ, Prinzen FW, Peeters RLM, Westra RL, Volders PGA. In Vivo Validation of Electrocardiographic Imaging. JACC Clin Electrophysiol [Internet]. 2017;3:232-242. Available from: https://linkinghub.elsevier.com/retrieve/pii/S2405500X16305151

15. Bear LR, LeGrice IJ, Sands GB, Lever NA, Loiselle DS, Paterson DJ, Cheng LK, Smaill BH. How Accurate Is Inverse Electrocardiographic Mapping? Circ Arrhythmia Electrophysiol [Internet]. 2018;11:e006108. Available from: http://circep.ahajournals.org/lookup/doi/10.1161/CIRCEP.117.0061 08

16. Sapp JL, Dawoud F, Clements JC, Horáček BM. Inverse solution mapping of epicardial potentials: Quantitative comparison with epicardial contact mapping. Circ Arrhythmia Electrophysiol [Internet]. 2012;5:1001-1009. Available from: http://circep.ahajournals.org/content/early/2012/08/24/CIRCEP.111. 970160.abstract

17. Duchateau J, Sacher F, Pambrun T, Derval N, Chamorro-Servent J, Denis A, Ploux S, Hocini M, Jaïs P, Bernus O, Haïssaguerre M, Dubois R. Performance and limitations of noninvasive cardiac activation mapping. Heart Rhythm [Internet]. 2018 [cited 2018 Oct 29];0. Available from: https://linkinghub.elsevier.com/retrieve/pii/S1547527118310269 
18. Sosa E, Scanavacca M, D’Avila A, Pilleggi F. A New Technique to Perform Epicardial Mapping in the Electrophysiology Laboratory. $J$ Cardiovasc Electrophysiol. 1996;7:531-536.

19. Coronel R, de Bakker JMT, Wilms-Schopman FJG, Opthof T, Linnenbank AC, Belterman CN, Janse MJ. Monophasic action potentials and activation recovery intervals as measures of ventricular action potential duration: Experimental evidence to resolve some controversies. Heart Rhythm [Internet]. 2006;3:10431050. Available from: http://dx.doi.org/10.1016/j.hrthm.2006.05.027

20. Orini $M$, Taggart $P$, Lambiase PD. In vivo human sock-mapping validation of a simple model that explains unipolar electrogram morphology in relation to conduction-repolarization dynamics. $J$ Cardiovasc Electrophysiol [Internet]. 2018;29:990-997. Available from: http://doi.wiley.com/10.1111/jce.13606

21. Orini M, Taggart P, Srinivasan N, Hayward M, Lambiase PD. Interactions between activation and repolarization restitution properties in the intact human heart: In-vivo whole-heart data and mathematical description. PLoS One [Internet]. 2016;11:e0161765. Available from: http://dx.plos.org/10.1371/journal.pone.0161765

22. Martin CA, Orini M, Srinivasan NT, Bhar-Amato J, Honarbakhsh S, Chow AW, Lowe MD, Ben-Simon R, Elliott PM, Taggart P, Lambiase PD. Assessment of a conduction-repolarisation metric to 
predict Arrhythmogenesis in right ventricular disorders. Int J Cardiol [Internet]. 2018;271:75-80. Available from:

https://linkinghub.elsevier.com/retrieve/pii/S0167527318320497

23. Ramanathan C, Rudy Y. Electrocardiographic imaging: II. Effect of torso inhomogeneities on noninvasive reconstruction of epicardial potentials, electrograms, and isochrones. J Cardiovasc

Electrophysiol. 2001;12:241-252.

24. Rudy Y. Noninvasive ECG imaging (ECGI): Mapping the arrhythmic substrate of the human heart. Int J Cardiol [Internet]. 2017;237:1314. Available from:

https://linkinghub.elsevier.com/retrieve/pii/S0167527317307556

25. Ghanem RN, Burnes JE, Waldo AL, Rudy Y. Imaging dispersion of myocardial repolarization, II: Noninvasive reconstruction of epicardial measures. Circulation. 2001;104:1306-1312.

26. Oster HS, Taccardi B, Lux RL, Ershler PR, Rudy Y. Noninvasive electrocardiographic imaging: reconstruction of epicardial potentials, electrograms, and isochrones and localization of single and multiple electrocardiac events. Circulation. 1997;96:10121024.

27. Ramanathan C, Jia P, Ghanem R, Calvetti D, Rudy Y. Noninvasive electrocardiographic imaging (ECGI): Application of the generalized minimal residual (GMRes) method. Ann Biomed Eng. 2003;31:981- 
994.

28. Revishvili AS, Wissner E, Lebedev DS, Lemes C, Deiss S, Metzner A, Kalinin V V., Sopov O V., Labartkava EZ, Kalinin A V., Chmelevsky M, Zubarev S V., Chaykovskaya MK, Tsiklauri MG, Kuck KH. Validation of the mapping accuracy of a novel noninvasive epicardial and endocardial electrophysiology system. Europace. 2015;17:1282-1288.

29. Tung R, Josephson ME, Bradfield JS, Shivkumar K. Directional Influences of Ventricular Activation on Myocardial Scar Characterization: Voltage Mapping with Multiple Wavefronts during Ventricular Tachycardia Ablation. Circ Arrhythmia Electrophysiol [Internet]. 2016;9. Available from:

http://circep.ahajournals.org/content/9/8/e004155.long

30. Glashan CA, Androulakis AFA, Tao Q, Glashan RN, Wisse LJ, Ebert M, De Ruiter MC, Van Meer BJ, Brouwer C, Dekkers OM, Pijnappels DA, De Bakker JMT, De Riva M, Piers SRD, Zeppenfeld K. Whole human heart histology to validate electroanatomical voltage mapping in patients with non-ischaemic cardiomyopathy and ventricular tachycardia. Eur Heart J [Internet]. 2018;39:28672875. Available from:

https://academic.oup.com/eurheartj/article/39/31/2867/4956395

31. Cluitmans M, Brooks DH, MacLeod R, Dössel O, Guillem MS, Van 
Dam PM, Svehlikova J, He B, Sapp J, Wang L, Bear L. Validation and opportunities of electrocardiographic imaging: From technical achievements to clinical applications. Front Physiol [Internet]. 2018;9:1305. Available from:

https://www.frontiersin.org/article/10.3389/fphys.2018.01305/full

32. Tschabrunn CM, Roujol S, Dorman NC, Nezafat R, Josephson ME, Anter E. High-Resolution Mapping of Ventricular Scar: Comparison between Single and Multielectrode Catheters. Circ Arrhythmia Electrophysiol [Internet]. 2016;9. Available from:

https://www.ahajournals.org/doi/10.1161/CIRCEP.115.003841 


\section{Tables}

Table 1: Baseline Characteristics. IHD - Ischaemic heart disease, DCM - Dilated Cardiomyopathy, ARVC - arrhythmogenic right ventricular cardiomyopathy, BrS Brugada syndrome, Ao - Aorta, RV - right ventricular, RVOT - right ventricular outflow tract, A - Atria.

\begin{tabular}{|c|c|c|c|c|c|c|c|}
\hline Patient & Age & Sex & Aetiology & Rhythm & $\begin{array}{c}\text { Anatomical } \\
\text { structure }\end{array}$ & $\begin{array}{c}\text { UEG } \\
\text { CARTO } \\
\text { signals }\end{array}$ & Pacing maps \\
\hline 1 & 46 & F & ARVC & RV pacing & RVOT & 6289 & No \\
\hline 2 & 73 & M & IHD & Bi-V pacing & Aorta & 3007 & Yes \\
\hline 3 & 48 & M & BrS & RV pacing & Aorta & 2614 & Yes \\
\hline 4 & 24 & F & ARVC & RV pacing & RVOT & 5755 & No \\
\hline 5 & 43 & M & N/A & RV Pacing & Aorta & 6610 & No \\
\hline 6 & 52 & F & ARVC & RV pacing & RVOT & 1077 & Yes \\
\hline 7 & 58 & M & ARVC & RV pacing & RVOT & 4573 & Yes \\
\hline 8 & 21 & M & DCM & A pacing & Aorta & 619 & No \\
\hline
\end{tabular}


Table 2. Agreement between ECGI and contact mapping. $\mathrm{N}-\mathrm{el}=$ number of bodysurface electrodes used for ECGI calculation; Pairs: Number of epicardial points with simultaneous ECGI and CARTO data. $\rho_{A T}$ and $\rho_{R T}$ : Correlation coefficients for activation and repolarization times; $E_{A T}$ and $E_{R T}$ : Root mean square error for activation and repolarization times; $, \rho_{Q R S}^{\text {med }}, \rho_{T W}^{\text {med }}$ and $\rho_{U E G}^{\text {med }}$ : Morphological correlation coefficient measured within the QRS, T-waves and entire signals, respectively. UEG = Unipolar electrogram. $\mathrm{SD}=$ Standard deviation, $\mathrm{Q}=$ quartile, $\mathrm{n}=$ number, $\mathrm{n} . \mathrm{u} .=$ normalized units.

\begin{tabular}{|l|ccc|cc|cc|ccc|}
\hline Patient & $\begin{array}{c}\text { N-el } \\
(n)\end{array}$ & $\begin{array}{c}\text { QRS } \\
(m s)\end{array}$ & $\begin{array}{c}\text { Pairs } \\
(n)\end{array}$ & $\begin{array}{c}\rho_{\mathrm{AT}} \\
(\text { n.u. })\end{array}$ & $\begin{array}{c}\rho_{\mathrm{RT}} \\
(\text { n.u. })\end{array}$ & $\begin{array}{c}E_{A T} \\
(m s)\end{array}$ & $\begin{array}{c}E_{\mathrm{RT}} \\
(m s)\end{array}$ & $\begin{array}{c}\rho_{Q R S}^{\text {med }} \\
(\text { n.u. })\end{array}$ & $\begin{array}{c}\rho_{T W}^{\text {med }} \\
(\text { n.u. })\end{array}$ & $\begin{array}{c}\rho_{U E G}^{\text {med }} \\
(\text { n.u. })\end{array}$ \\
\hline 1 & 207 & 155 & 890 & 0.76 & 0.72 & 25 & 62 & 0.68 & 0.45 & 0.71 \\
2 & 218 & 153 & 727 & 0.67 & 0.59 & 45 & 78 & 0.59 & 0.44 & 0.61 \\
3 & 201 & 164 & 584 & 0.80 & 0.50 & 24 & 55 & 0.67 & 0.80 & 0.69 \\
4 & 175 & 95 & 919 & 0.65 & 0.69 & 23 & 29 & 0.75 & 0.71 & 0.78 \\
5 & 179 & 110 & 926 & 0.51 & 0.33 & 27 & 46 & 0.60 & 0.69 & 0.71 \\
6 & 172 & 101 & 232 & 0.69 & 0.84 & 18 & 19 & 0.78 & 0.80 & 0.78 \\
7 & 175 & 123 & 694 & 0.29 & 0.30 & 36 & 103 & 0.24 & -0.11 & 0.23 \\
8 & 181 & 98 & 285 & 0.54 & 0.51 & 15 & 48 & 0.78 & 0.26 & 0.71 \\
\hline mean & 189 & 125 & 657 & 0.62 & 0.56 & 27 & 55 & 0.63 & 0.51 & 0.65 \\
SD & 18 & 28 & 274 & 0.16 & 0.19 & 10 & 27 & 0.18 & 0.32 & 0.18 \\
\hline First Q & 175 & 100 & 435 & 0.53 & 0.41 & 21 & 38 & 0.59 & 0.35 & 0.65 \\
Median & 180 & 117 & 711 & 0.66 & 0.55 & 24 & 51 & 0.67 & 0.57 & 0.71 \\
Third Q & 204 & 154 & 905 & 0.73 & 0.71 & 32 & 70 & 0.76 & 0.76 & 0.74 \\
\hline
\end{tabular}


Table 3: Distance between pacing site and earliest region of activation in ECGI maps. $\mathrm{N}=$ number of pacing sites; $\mathrm{Q}=$ quartile; $\mathrm{Med}=$ median; $\mathrm{S}-\mathrm{QRS}=$ Interval from stimulus to QRS

\begin{tabular}{|c|cccc|cccc|}
\cline { 2 - 9 } \multicolumn{1}{c|}{} & \multicolumn{4}{c|}{ Any S-QRS interval } & \multicolumn{4}{c|}{ S-QRS $<40 \mathrm{~ms}$} \\
\hline Patient & $\mathrm{N}$ & $\mathrm{Q} 1(\mathrm{~mm})$ & Med $(\mathrm{mm})$ & $\mathrm{Q} 3(\mathrm{~mm})$ & $\mathrm{N}$ & $\mathrm{Q} 1(\mathrm{~mm})$ & Med(mm) & Q3 $(\mathrm{mm})$ \\
2 & 4 & 0.0 & 6.1 & 15.0 & 4 & 0.0 & 6.1 & 15.0 \\
3 & 17 & 14.8 & 31.3 & 36.3 & 11 & 7.9 & 30.9 & 32.9 \\
6 & 8 & 8.5 & 22.5 & 35.5 & 3 & 3.4 & 13.6 & 23.4 \\
7 & 17 & 8.9 & 19.4 & 31.7 & 7 & 0.0 & 6.8 & 12.3 \\
\hline TOT & 46 & 9.6 & 20.7 & 33.2 & $\mathbf{2 5}$ & $\mathbf{0 . 0}$ & $\mathbf{1 3 . 2}$ & $\mathbf{2 8 . 3}$ \\
\hline
\end{tabular}




\section{Figures}
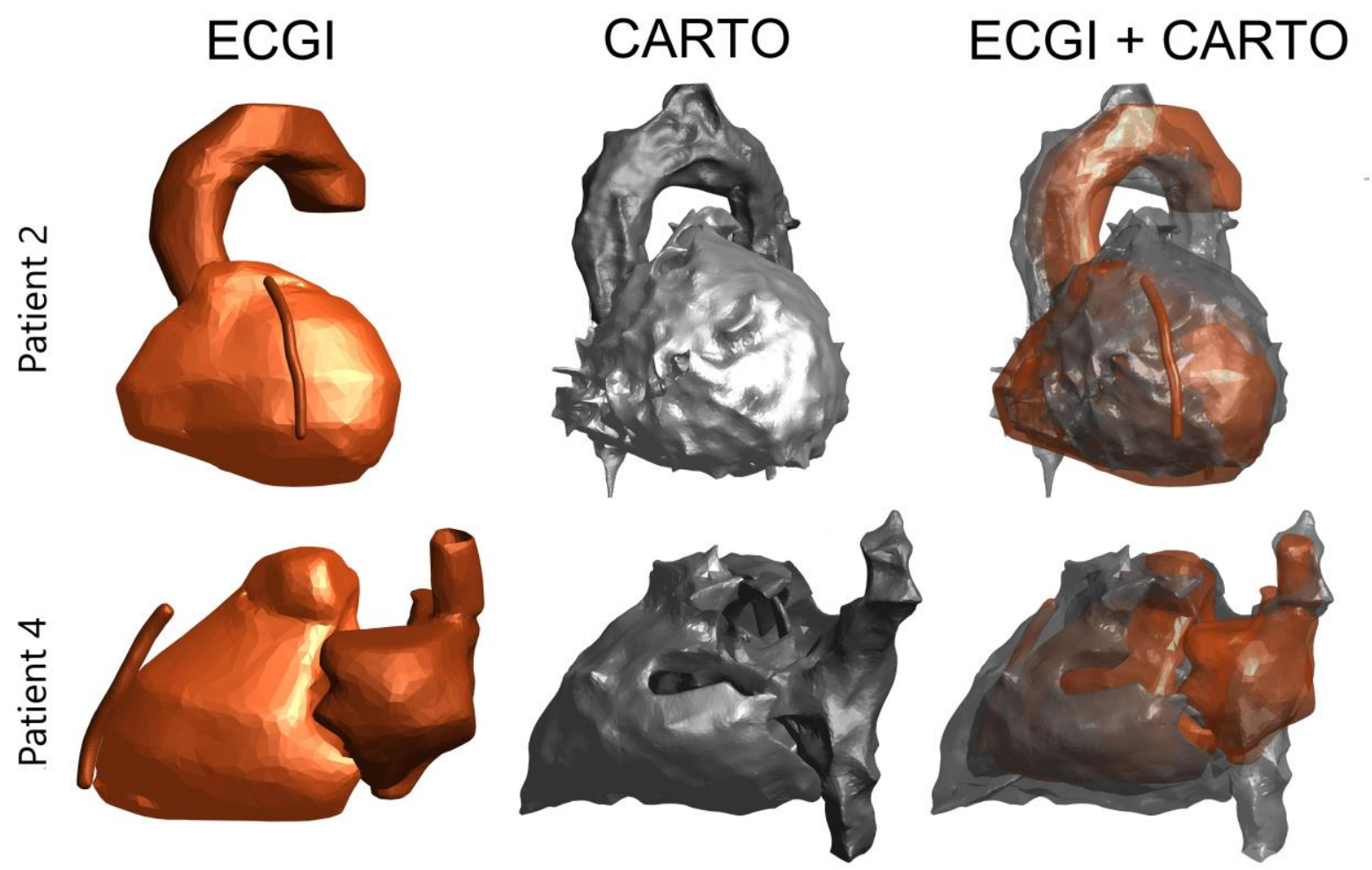

Figure 1: Anatomical coregistration of ECG imaging (ECGI) and CARTO geometries in 2 patients. ECGI and CARTO geometries are shown on the left and in the middle, respectively, and they are combined in a unified reference system on the right. Patient 2 is displayed in left anterior oblique and patient 4 in left lateral view. 

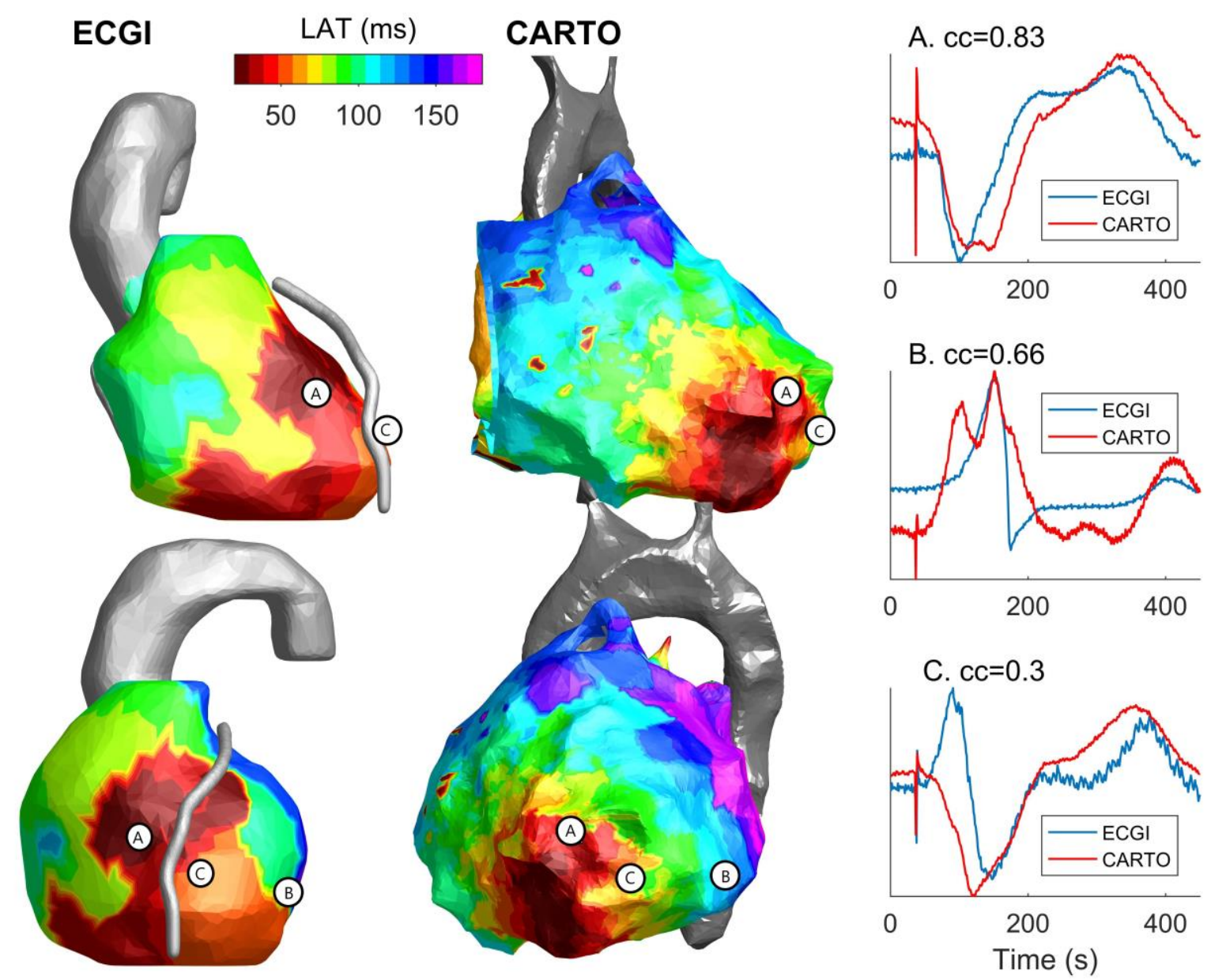

Figure 2. Comparison of activation times during right ventricular (RV) pacing for ECG imaging (ECGI) and CARTO. Images on top are seen in right lateral view and the bottom ones in left anterior oblique. Area of earliest activation is displayed in red with purple representing areas of latest activation. Labels A, B, and C on the geometry corresponds to sites where morphological similarity of QRS complexes is high (A, correlation coefficient $[\mathrm{cc}]=0.83,75$ th percentile of $\mathrm{ccs})$, good $(\mathrm{B}, \mathrm{cc}=0.66$, median correlation), and low ( $\mathrm{C}, \mathrm{cc}=0.30,25$ th percentile of $\mathrm{ccs})$. Unipolar electrograms are shown on the right $(\mathbf{A}-\mathbf{C})$. LAT indicates local activation time in milliseconds. 


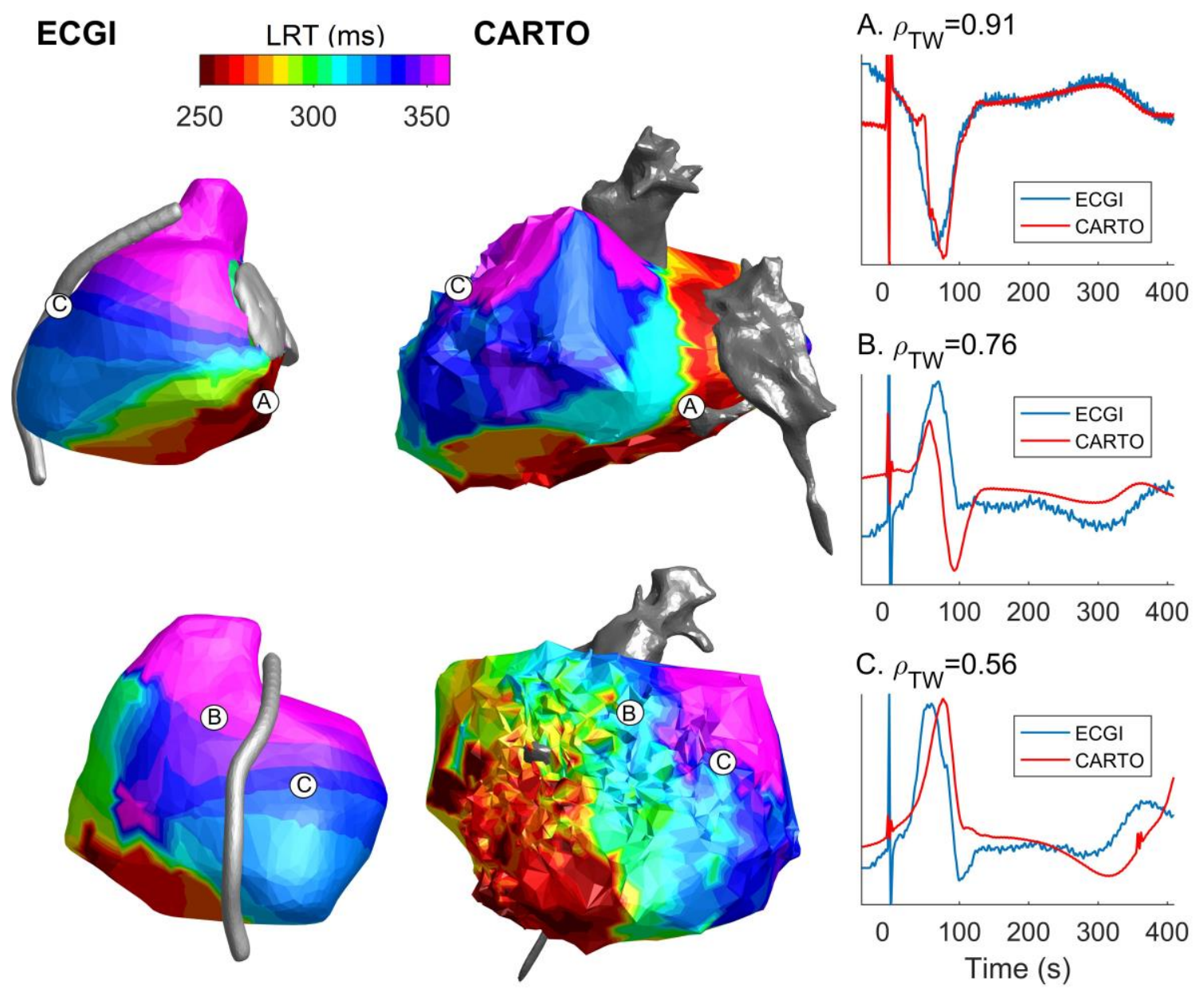

Figure 3. Comparison of repolarization time maps from ECG imaging (ECGI) and

CARTO in a representative patient. The top pictures are in left lateral view and the bottom in left anterior oblique. The left anterior descending artery is displayed for orientation and demarcation of left and right ventricles. Early repolarization is in red and late in purple. Labels $\mathrm{A}, \mathrm{B}$, and $\mathrm{C}$ on the geometry corresponds to sites where morphological similarity of the T-wave of the unipolar electrogram is high (A, correlation coefficient $[\mathrm{cc}]=0.91,75$ th percentile of $\mathrm{ccs})$, good $(\mathrm{B}, \mathrm{cc}=0.76$, median correlation), and low ( $\mathrm{C}, \mathrm{cc}=0.56,25$ th percentile of $\mathrm{ccs})$. Unipolar electrograms at these sites are shown on the right $(\mathbf{A}-\mathbf{C})$. LRT indicates local repolarization time in milliseconds. 


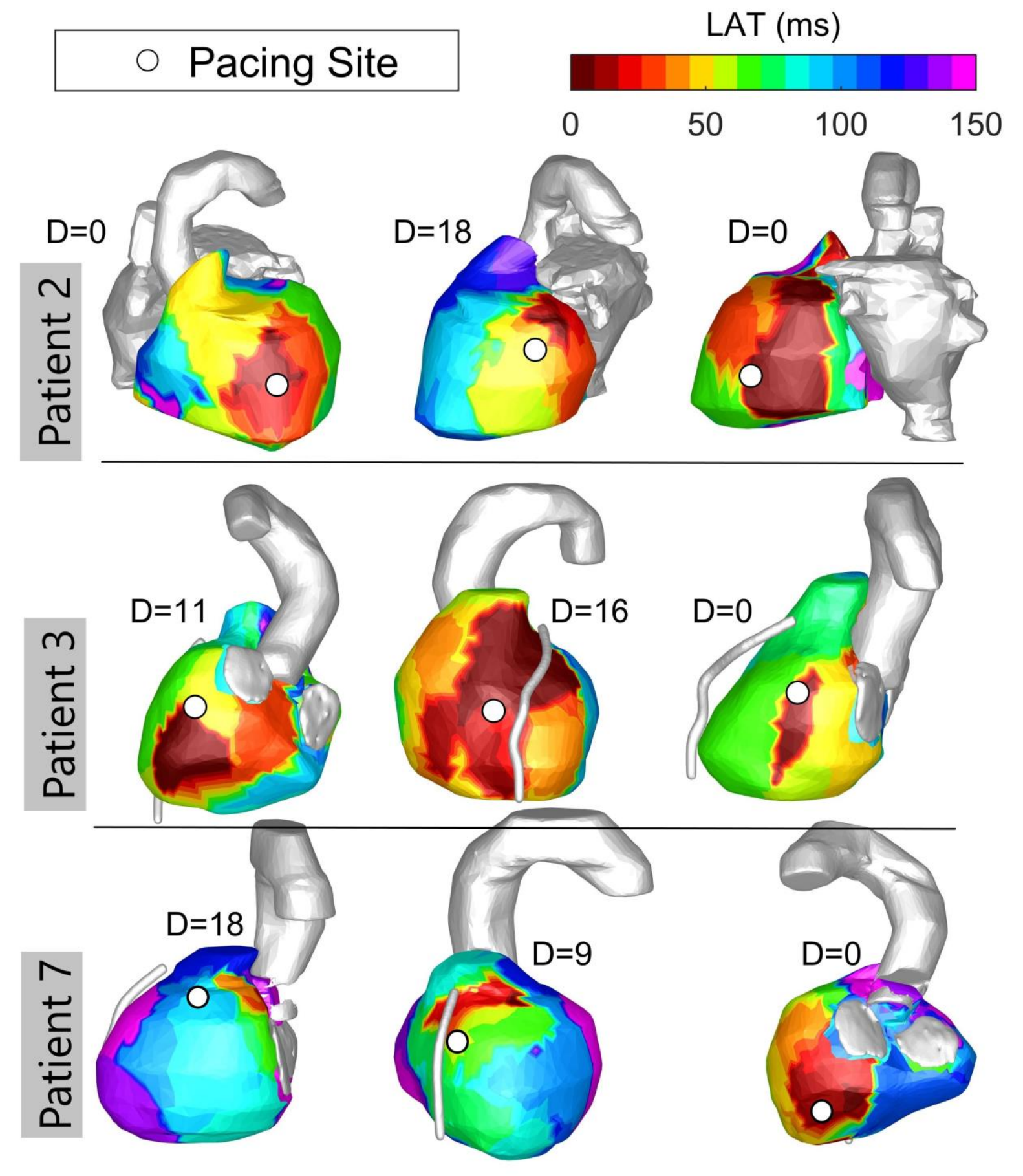

Figure 4. Relation between pacing site location as defined by CARTO position at time of pacing (white circles) and ECGI maps of activation time. Three patients are included with 3 pacing sites for each. Area of early activation is in red with late activation in purple. Distance between pacing site and region of earliest activation (D) is reported next to each map. LAT indicates local activation time in milliseconds. 